Carlos Keller $\mathbf{R}$.

\title{
El capitalismo primitivo
}

ARA ciertos economistas. nuestro sistema económico actual obedece a ciertos principios que consideran absolutos, eternos, insuperables. Creen que, en 1789. la humanidad alcanzó una altura que si bien no era perfecta del todo, se está perfeccionando rápidamente; de manera que todo el desarrollo histórico de los siglos pasados llega a culminar precisamente en nuestros dias.

Pero la evolución que hemos presenciado en los últimos años nos ha demostrado de nuevo cuán útil es considerar todos los fenómenos sociales desde el punto de vista histórico. Dentro de unos pocos siglos o quizás decenios. nuestro capitalismo llegará a pertenecer a la historia, y entonces los hombres sonreirán al leer las obras ingenuas que han publicado nuestros hombres progresistas.

En realidad, el capitalismo moderno es un sistema económico que ya está adquiriendo caracteres históricos en muchas partes. para ser superado y sustituido por otros organismos económicos que no están determinados por los principios fundamentales del capitalismo. Citemos como ejemplo el principio de la libre competencia. En los grandes etrusts,. propietarios hoy de numerosas. importantes materias primas, como ser el petróleo. el cobre, el yodo. etc., y de numerosos grupos de las grandes industrias, ya no está en vigencia aquel principio. La economía de los últimos años nos presenta en todas partes la formación 
de tales organismos de enormes proporciones que quizás llegarán a organizar algún dia toda la economia mundial sobre la base de un único sistema económico. Esos orģanismos tienen gran semejanza con el ideal económico que preconcibió Carlos Marx: se trata de sustituir la libertad casi ilimitada del capitalismo moderno por una organización de la economía en la forma de enormes trusts. los que pueden llamarse estados, y dentro de los cuales cada obrero y empleado tiene la calidad de un empleado fiscal.

Pero quizás sea prematuro hablar de esta evolución que apenas comienza a manifestarse. Cierto es, con todo. que el optimismo que reinaba hace sólo unos pocos decenios respeclo del capitalismo moderno. se está desvaneciendo. A la nueva generación, se le presenta como algo que ya se está convirtiendo en historia. Ya no nos encontramos en el centro de la evolución capitalista moderna; podemos observar sus fenómenos en una forma más desapasionada, más tranquila. Y quizás, como consecuencia de ello. las épocas que precedieron al capitalismo moderno. y que fueron combatidas tan enfáticamente por el capitalismo, ya no se nos manifiestan en esa forma lúgubre. feroz. como a nuestros padres. Estamos en situación de contemplarlas como meros observadores.

No digo yo con esto que la época que precedió directamente al capitalismo moderno haya desaparecido del todo. Por el contrario. la mayoría de los Estados se encuentra aún en gran parte en la época que denominaré del capitalismo primitivo. Aun en nuestro propio país, los fundamentos del capitalismo primitivo están en vigencia en varias ramas de la economía y sọn aún la forma de vida de vastas clases sociales.

Para un investigador moderno, existen tres diferentes capas económicas simultáneas en nuestra economia actual: el capitalismo primitivo. el capitalismo moderno y aquellas primeras manifestaciones de una nueva evolución económica, todavía no denominada.

El capitalismo primitivo es aquella forma de satisfacer las neøesidades humanas que llegó a desarrollarse desde que España 
se colocó a la cabeza de las naciones occidentales; es decir. desde el tiempo de Carlos V. y. especialmente, de Felipe II. cuyas reformas económicas no han sido estudiadas hasta la fecha en la forma que merecen. La primera nación que dió una nueva organización económica a los pueblos occidentales. fué. Inglaterra. desde mediados del siglo XVIII. Pronto la siguió Francia.

En Alemania, la nueva evolución-que llamaremos el capitalismo moderno-sólo llegó a manifestarse después de la revolución del año 48. En Estados Unidos de Norte América llegó a predominar después de la Guerra de los Esclavos, y en la mayoría de las demás naciones, en una fecha posterior.

Imposible es fijar fechas absolutas. Los grandes movimientos sociológicos se producen lentamente. y como ya dije. las formas pre-existentes no desaparecen desde luego totalmente, sino se mantienen, perdiendo, si. parte de su influencia.

Trataremos de exponer aqui en grandes rasgos, en qué consiste el capitalismo primitivo, comparado con el capitalismo moderno.

En la época del capitalismo primitivo. el hombre era aún la medida de todas las cosas.

Es éste uno de los principios fundamentales que estaban en vigencia hasta principios del siglo actual. Interesante es observar las luchas que se libraron en aquel tiempo sobre esta cuestión. Newton era uno de los primeros genios que preconcibieron las ciencias modernas. Empleaba aparatos cientificos para observar los fenómenos de la naturaleza: definía los colores según las ondulaciones del rayo de luz. De esta manera. se llega a fijar la existencia de colores invisibles para nuesíros ojos. como, p. ej.. el color ultravioleta. Goethe. genio quizás mayor que Newton, también estudió los colores y publicó una obra de gran alcance sobre ellos. En su controversia con Newton, niega la existencia de colores invisibles para nuestros ojos. Para él, el universo sólo existe en cuanto puede ser observado 
por los sentidos del hombre. No reconoce la existencia de fenómenos físicos fuera de lo que puede observar el hombre: el hombre es la medida de todas las cosas.

Pero el hombre no sólo es la medida de las cosas, sino que se encuentra, también, en el centro de la economía. En el capitalismo primitivo, el negocio sirve al hombre, $y$ no el hombre al negocio. El hombre no era aún el esclavo de la economía. Era considerada ésta como mero medio necesario para poder existir. En el centro de la preocupación de los hombres se encontraba aún... su alma. su espíritu. La religión. las artes, la filosofia. las diversiones de toda especie. Ilenaban la mayor parte de las horas del día. El ideal consistia en la perfección de la parte espiritual, eterna del hombre; no en tesorar riquezas, en producir bienes materiales.

Asi se explica que los hombres del siglo XVIII fueran hombres perezozos. para emplear un calificativo moderno. Franklin. una de las grandes personalidades de la época. y. además, uno de los hombres más laboriosos, dice en sus Memorias, que su jornada de trabajo diaria era de seis horas. Y se consideraba como excepcionalmente trabajador. En la mayoría de las pequeñas industrias de esa época, el trabajo cesaba durante muchos meses del año. No habia trabajo para ocupar a los trabajadores. como, p. ej.. no lo hay durante todo el año en numerosisimos fundos de nuestro pais, en el siglo $\mathrm{XX}$.

Todas las actividades se ejecutaban con gravedad y reposo. No se conocia aún esa manera. tan peculiar a nuestras grandes urbes, de moverse y de Irabajar. Aun hoy. un provinciano pierde fácilmente la razón al tener que cruzar una calle central en cualquiera ciudad de más de 200.000 habitantes. $\mathrm{Y}$ colóquese en nuestras calles modernas a un grandseigneur del rococó: no podria moverse en este mundo tan atolondrado y extraño.

A este ideal de una vida tranquila y reposada, correspondian las máximas de la vida comercial. Trabajar lo menos posible y hacer grandes ganancias. era el ideal del comercio, en oposición al moderno. el cual, si bien es obtener las mayores ganancias posibles, trata de hacerlas mediante grandes transacciones y 
poca ganancia por unidad. En el siglo XVI. los comerciantes de Sevilla consideraban un $300 \%$ como ganancia mezquina.

Al mismo principio obedece la resistencia que se manifiesta contra la libre competencia. Hoy están permitidos todos los medios, fuera de las pocas prohibiciones que contiene el Código Penal. En el período del capitalismo primitivo existian numerosísimas trabas que se oponían a ese principio. Era prohibido. por ejemplo, hacer réclame de cualquiera especie. En Paris, se prohibieron, a fines del siglo XVIII. los avisos en los periódicos.

Toda la vida era tradicionalista. Lo que habian considerado razonable los padres y los abuelos era aplicado. tanto en cuanto a los métodos técnicos. como en cuanto a las máximas éticas que regian la vida económica. El pasado domina completamente sobre el presente, mientras que en la época del capitalismo moderno basta con expresar que un método técnico. p. ej.. data de hace 10 o 20 años para descalificarlo completamente. Esta forma de concebir la vida fué modificada sólo a fines del siglo XVIII, cuando se inició el movimiento racionalista. que es. en cierta manera, una de las bases del capitalismo moderno. Era uno de los grandes golpes contra el empirismo que había predominado hasta entonces.

Debido a este tradicionalismo. las bases de la economía eran muy sólidas y estables. No se conocian aún las grandes crisis económicas del siglo XIX. Cierto es que hubo crisis en todos los tiempos, pero estaban ellas. antes del siglo XIX. basadas en catástroles extraordinarias, como las grandes sequias, las guerras, efc., y no en meros hechos económicos, como las expeculaciones erróneas.

Existía una ética bien definida sobre muchos hechos económicos. los precios. los intereses que era lícito cobrar. etc.. máximas que se habian conservado desde la Edad Media y que vinieron a desaparecer en el curso de la evolución del capitalismo moderno. 
Existía, en la época del capitalismo primitivo, un sistema económico bien organizado. La legislación económica estaba mucho más desarrollada que en nuestros días. En el campo, habia comunidades rurales que regularizaban toda la vida campesina. La propiedad rústica estaba parcelada en pequeñas fajas de terrenos que no se podian explotar, sino mediante un trabajo sistemático y ordenado de todos los vecinos. En consecuencia, existian reglamentos sobre todas las faenas. El tiempo de la siembra, de la cosecha, de la preparación de los campos, era fijado por la comunidad. Dentro de las ciudades, correspondía igual importancia a los gremios. El maestro del gremio repartía los trabajos entre los diferentes artesanos. prescribia los materiales que se debian emplear. fijaba el número de aprendices y de oficiales que podian ocuparse.

El ideal social de esta organización consistia en una democracia de hecho, y no formal, como lo es la nuestra. El fin de toda la legislación económica consistía en impedir la formación de grandes fortunas. Quien trataba de quitar su clientela a un artesano. era castigado rigurosamente. Hacerle posible la vida a la mayor cantidad de hombres de igual situación social y de iguales rentas, era uno de los principios fundamentales de este período.

Este ideal fué destruido por el capitalismo moderno, mediante un sistema de privilegios concedidos por los Estados a ciertos empresarios. Obtuvieron éstos el derecho de ponerse por encima de todas esas trabas que existian para los demás hombres. Estos primeros empresarios eran modernos piratas politicos, que habian reunido una fortuna en forma más o menos aventurera, y que se dedicaron. con sus riquezas, a la producción. Sombart, en su soberbia obra .El capitalismo moderno, cita numerosos ejemplos a este respecto. En su obra, el lector encontrará documentados minuciosamente los hechos a que me refiero aquí. 
No menos sujeta a un sinnúmero de disposiciones legales estaba también la técnica en el periodo del capitalismo primitivo. Como ya hemos visto, predominaba lo empírico. No se empleaban aún los mélodos mecánicos y cientificos de que disponen las empresas modernas. Estaba limitada la técnica capitalista primitiva por los limites de la naturaleza orgánica. El hombre empleaba casi exclusivamente materiales de la naturaleza orgánica. Por ejemplo, en primer lugar. la madera. Casi todos los utensilios y herramientas eran de madera. También se empleaba esta materia para producir otros materiales. como el fierro: los hornos eran calentados con leña. En la tintorería y en la mayor parte de las industrias, se empleaban materias de la naturaleza orgá. nica. Y en vez del vapor que mueve nuestras fábricas, la energía del capitalismo primitivo era el agua de los ríos y arroyos y el viento. El molino es el establecimiento industrial peculiar a este periodo. y asi lo vemos predominar también en el paisaje.

Las relacionés económicas entre los individuos tenían un carácter netamente personal: todos los negocios se cerraban por los mismos interesados: no se conocía la compra a grandes distancias. ni tampoco la compra por muestras; en las grandes ferias de este periodo. las mercaderias estaban a la vista.

El mismo carácter presentan los créditos y aún los empréstitos fiscales. los cuales eran facilitados a los Estados por grandes capitalistas. No se conocian todavia los bonos hipotecarios: las letras no eran endosadas: el sistema monetario era rigido. y no se conocía el papel moneda. Tampoco les correspondía importancia alguna. dentro de la economia, a las sociedades anónimas, con sus acciones al portador, propias del capitalismo moderno. Cada empresa pertenecía a un determinado in. dividuo conocido de todo el mundo. mientras que en nuestre 
economía, nadie sabe a beneficio de quièn trabaja y produce una empresa.

Este mismo carácter personal existía también en cuanto a las relaciones entre el patrón y el obrero. El patrón se consideraba artesano llegado a mayor fortuna, debido a las contingencias de la vida: no pertenecia a una casta social separada por todo un mundo del obrero. La familia patriarcal se mantenia intacta, y el hogar comprendia también a los domésticos y oficiales empleados en la respectiva industria.

La base de toda la economia era la agricultura. Según un censo levantado en Francia en 1818. el $79 \%$ de la población era rural. En Alemania, aun en 1849. la población rural formaba el $61 \%$ de la población del pais: actualmente no alcanza al $30 \%$.

Los paises europeos eran exportadores de cereales y otros productos agrícolas. A pesar de su población relativamente elevada, habia un sobrante en la agricultura; mientras que en nuestros dias depende su existencia de la importación de cereales y carnes extranjeras.

En el curso del siglo XVIII, y aun antes en ciertas regiones y en algunas ramas de la economía. como luego veremos. este sistema económico experimenta un completo trastorno. Las verdaderas causas de este nuevo movimiento son de indole sociológica, y no vamos a analizarlas en este estudio. Podemos decir, si, que dentro de la economia, se nos manifiesta un nuevo espiritu: si antes el hombre, con sus anhelos espirituales superiores. se habia encontrado en el centro de todas las actividades sociales, de aqui en adelante, él ya no trabaja con el exclusivo fin de ganarse la vida. sino con el objeto de obtener ganancias, de dar a sus actividades económicas el mayor desarro- 
llo posible y de imponerse en la lucha por la vida económica. La economia comienza a observar completamente fodas las demás actividades humanas.

Este nuevo desarrollo,--que conduce hacia el capitalismo moderno,-comienza en el comercio al por mayor. En las ciudades italianas del período gótico. ya habian existido grandes empresas mercantiles que pueden calificarse de capitalistas, en el sentido en que empleamos este término actualmente. Allí se desarrolla, por primera vez en Occidente, un sistema monetario bien organizado. Alli comienza a independizarse el sistema económico de la voluntad del hombre, y a imponer a ésta sus leyes propias. Alli todos los hechos económicos llegan a ser reducidos a números que representan valores y que forman una de las mayores maravillas espirituales que haya producido el genio occidental: la contabilidad. En la contabilidad moderna. se reflejan todos los hechos económicos de la empresa. Aun más: todos aquellos hechos sólo se realizan con el objeto de producir un saldo lo más favorable posible para el capitalista. La contabilidad llega a ocupar una situación central dentro de nuestra economía. Y. simultáneamente, tiene lugar el desarrollo de la empresa dirigida a la autonomia del individuo a que pertenece. En el capitalismo moderno, el hombre desempeña un papel secundario dentro de la economia: es la empresa. como tal. la que le impone su voluntad; el hombre es el esclavo de su negocio. Por eso hablamos con mucha razón de la firma o razón social como de un ser independiente de su propietario: puede adquirirse y venderse esa firma social.

Esas primeras grandes casas comerciales fueron pronto superadas por las Sociedades Coloniales de Holanda e Inglaterra. Ellas iniciaron la compra a distancia y por muestras.

En las industrias se inició esta nueva evolución debido a ciertas condiciones naturales que requerían nuevos métodos para que se pudiera obtener un resultado. En la minoría disminuía la ley de los minerales extraidos de la tierra. y era necesario. en muchos casos. construir socavones y piques mucho más profundos, para poder explotar las minas. Ambas causas de- 
mandaban la inversión de capitales de que no disponian los pequeños artesanos. Preséntase, entonces, un capitalista que ofrece este capital al minero. En los primeros tiempos, éste lo acepta en calidad de préslamo: pero, cuando continuaba careciendo de capital o cuando cualquier percance le hacia imposible el pago del capital e intereses, el capitalista le embargaba la mina y comenzaba a explotar por su propia cuenta. Haciase fambién necesario emplear un mayor número de obreros: $y$ he ahi a nuestro capitalista moderno con la propiedad de la mina, haciendo trabajar bajo sus órdenes a 50.50 o más mineros.

En las demás industrias, puede observarse la misma evolución hacia el capilalismo. Los tejedores no estaban en situación de poder comprar las materias primas necesarias; viene un capilalista y se las facilita. Respecto del sistema económico de producir. nada ha cambiado por el momento: los artesanos continúan trabajando en sus pequeños talleres propios, pero producen pronto por cuenta exclusiva del capitalista. En una época posterior, éste comienza a establecer un taller propio y obliga a los artesanos a prestarle sus servicios en él. Más tarde aún, empléanse máquinas en grande escala, y el artesano independiente de antaño se convierte en obrero industrial.

Ese orden económico, como existía hasta principios del siglo $\mathrm{XIX}$, es decir, el trabajo de pequeños arlesanos que producen en su propio hogar. pero que ya dependen de un capitalista. nos es pintado en todos sus detalles en el tercer libro de la hermosisima novela de Wolfgang Goethe, Wilhelm Meister.

En este mismo periodo, existian también grandes industrias. pero no en la forma de empresas fabriles, sino de manufacturas. En ellas predominaba la obra de mano sobre la obra mecánica de la fábrica. Pero había también algunas fábricas. cuyo desarrollo data de mediados del siglo XVIII. La industria moderna no es precisamente la sucesora de la manufactura: ambas formas de organización representan, por el contrario. algo perfecto en sí y no superable. La muebleria artística moderna jamás puede ser sustituida por la fábrica, pues en ella siempre pre- 
dominará la obra de mano. Y de otro lado, muchas industrias modernas jamás estuvieron organizadas en forma de manufacturas.

Pero, a pesar de su relativa extensión, estas manufacturas e industrias no pertenecen al capitalismo desarrollado. Tenían un carácter rural; no había todavía centralización en grandes centros industriales: se producía en todas partes. pero especialmente en la vecindad de los ríos y bosques.

Hay que agregar a estos hechos, que fueron de tanta importancia para la formación del sistema económico aclual, otra circunstancia que fué, hasta cierto grado. casual. Me refiero a la expansión colonial. En las colonias, el capitalismo moderno se pudo desarrollar en una forma mucho más libre y vigorosa. porque no existian todas aquellas trabas a que me he referido. Con la ayuda de razas extranjeras. los colonizadores europeos se dedicaron a explotar las minas y a cultivar los campos. El europeo era aquí netamente el director de la empresa: los obreros eran siervos o semi-siervos. Asi se explica que con la institución de la servidumbre personal, de la mita, etc..-que existían tanto en las colonias españolas. como en todas las demás.- -se pudieran desarrollar empresas que son, en un alcance mucho mayor. los primeros representantes del capitalismo moderno. Aqui hubo minas con muchos centenares de obreros, y latifundios explotados según un plan preconcebido por el terrateniente.

Este nuevo tipo capitalista no se desarrolló en todas partes en la misma forma. En el imperio español existia un poder central vigoroso, y por eso, en las colonias españolas. gobernaba el representante del rey, quien imponía, en su calidad de tal, numerosas leyes sociales al colonizador. En las colonias holandesas e inglesas, en cambio, el poder se encontraba en manos del Director de la Compañia Colonizadora. Véase. por ejemplo, el caso de Lord Clive. 
Si bien en este periodo de nuestro desarrollo económico occidental hay manifestaciones de nuevas tendencias, no debe olvidarse que éstas son de poca intensidad. No existen aún resultados definitivos. Hay. en el mejor de los casos, una mezcla de dos diferentes sistemas. La causa de no haberse desarrollado formas puras, hay que buscarla en los embarazos de diferente indole que se oponian a la evolución.

Estos obstáculos, estas trabas, son, en primer lugar, de indole psicológica. Siempre es el hombre el causante de los grandes movimientos sociales. De la disposición de su espíritu depende. pues, más que de cualquiera otra causa, el resultado a que se llega. Y precisamente la disposición general del hombre en el período barroco, era opuesta a una plena evolución económica. Dedicar todas sus actividades a la economía. era cometer un sacrilegio, un pecado mortal. Se detestaba el exceso en el trabajo. El medio ambiente de aquel tiempo tenía un carácter rústico, rural. Predominaba la idiosincrasia del campesino, es decir. el conservatismo. la lentitud, la poca aggilidad espiritual. Las mejores fuerzas de la nación se ocupaban en actividades noeconómicas: las letras, las artes, la iglesia, la política, la administración, el ejército. AA iglesia, o mar, o casa real, dice un adagio del tiempo de Cervantes. Iglesia y casa real eran ocupaciones espirituales y administrativas; mar era. en primer lugar. la guerra marítima y la piratería. Las grandes familias vivian en la metrópoli y llevaban ahi una vida de rentistas, de lujo y diversiones. Las fincas eran administradas por empleados o dadas en arriendo.

El capital disponible en aquellos Estados barrocos no era invertido en la economía. sino, en su mayor parte, en empréstitos fiscales. El Fisco se encontraba en continuas penurias, pues se mantenían casi todas las naciones en no interrumpido estado de guerra. De los 200 años trascurridos desde 1600 hasta 1799. 154 corresponden a años de guerra en Europa. Y esas guerras 
eran. en gran parte, guerras a sangre y fuego. en que se destruian sistemáticamente vastas regiones productivas. No mencionaré sino la invasión de las provincias renanas hasta Heidelberg. por Luis XIV. quien ordenó destruir sistemáticamente todas las grandes ciudades de aquella región. de manera que el viajero que la visita no ve, aún hoy. sino ruinas de aquel tiempo a ambos lados del Rhin y en las regiones limitrofes. Además, se produjeron varias revoluciones en la segunda mitad del siglo XVIII. que igualmente estorbaron el desarrollo económico.

El fanatismo religioso no se tranquilizaba antes de expulsar de algunos Estados a grandes masas de incrédulos. Asi fueron expulsados de Francia los hugonotes, que se radicaron en Inglaterra y Alemania.

Otro grupo de embarazos que se oponía al desarrollo industrial. es uno de indole técnica. A él pertenece también la higiene deficiente: la peste se repetía con cierta regularidad cada diez años en Europa. causando enormes estragos en la población: el estado de salubridad de las grandes ciudades era pésimo, superando casi siempre las defunciones a los nacimientos.

Los procedimientos técnicos estaban aún muy poco desarrollados, por lo cual, y debido a la falta de materias primas. la producción no podia realizarse en grande escala. Faltaban especialmente maderas, porque las existencias forestales de la Europa occidental se habian agotado. Especialmente en los paises más adelantados de este período, es decir. en España. Italia y Francia, la falta de madera era muy grande. Hasta nuestros días. estos paises no poseen aún plantaciones forestales de alguna consideración. Así se explica también la tendencia de la política de estos paises a formar alianzas con los paises de la Europa oriental. los cuales les podían proporcionar las maderas necesarias. Y, como habíamos visto ya más arriba. la madera era en aquel tiempo la materia prima más importante (la producción de fierro era, en Inglaterra. de 1.700 ts. a 1.750). 
Todas estas causas explican un malestar social que se manifestaba en todas partes y que tuvo por consecuencia nuevos grandes trastornos sociales que dieron a la economia occidental una base completamente diferente. 